\title{
XIII.
}

Aus dem pharmakologischen Institut von Prof. v. Schroeder in Heidelberg.

\section{Ueber die Wirkungen des Tropins und der Tropeïne.}

Von

\author{
Privatdocent Dr. R. Gottlieb,
}

Assistent des Instituts.

(Mit 3 Carven.)

Mit dem Atropin werden bekanntlich eine Reihe ktinstlicher Ester des Tropins in die chemisehe Gruppe der Tropein ze zusammengefasst, in denen der im Atropin mit der Base verbundene Rest der Tropasäure durch andere Säureradicale ersetzt ist. In dem pharmakologischen System werden diese künstlichen Tropeïne seit Buchheirm dem Atropin an die Seite gestellt und mit einer Reihe natürlich vorkommender Alkaloide, in denen die Tropasäure mit dem Tropin isomeren Basen verbunden ist, zur Gruppe des Atropins und der Tropeïne vereinigt. Für die Pharmakologie bietet diese Gruppe die Aufgabe, die physiologischen Wirkungen der Tropeïne mit jenen des Atropins zu vergleichen und die Veränderungen kennen zu lernen, welche die Wirkung der Ester durch den Eintritt verschiedener Säureradicale aus der aliphatischen und aromatischen Reibe erleidet. Schon Buchheim I) bat auf diese Aufgabe hingewiesen und durch die Darstellung des Benzoyltropins und seine pharmakologische Vergleichung mit dem Tropin die in dieser Beziehung wohl bedeutungsvollste Thatsache ermittelt, dass das der Pupille gegenüber unwirksame Tropin erst durch die Verbindung mit dem aromatischen Säurerest mydriatische Wirkung erlangt. Obgleich bald darauf die Untersuchungen Ladenburg's ${ }^{2}$ ) eine bequeme Methode zur Darstellung der Tropeïne kennen gelehrt hatten, liegen aber über die Wirkung der seither - insbesondere von Ladenburg - dargestellten Tropeïne nur spärliche Notizen vor. Nur das Phenylglycolsäure-Tropin

1) Ueber die pharmakologische Gruppe des Atropin. Archiv f. exp. Path. u. Pharm. Bd. V.

2) Liebig's Annalen. Bd. CCVI. 
(Homatropin) hat durch seine praktische Anwendung Beachtung gefunden, während die übrigen Tropeïne meist nur auf ihre Pupillenwirkung geprüft, aber nicht näher pharmakologisch charakterisirt sind. Ich ergriff deshalb gern die Gelegenheit, die Wirkung einiger neuer Tropeïne zu untersuchen, welche mir durch die Freundlichkeit der chemischen Fabrik E. Mer ck in Darmstadt zur Verfügung standen. Ueber die Darstellung dieser Tropeïne hat Herr Merck in seinem Jahresberichte ïber das Jahr 1894 bereits Näheres mitgetheilt. Ich erhielt als schön krystallinische Präparate Acetyltropin, Succinyltropin, Lactyltropin und Hippuryltropin und habe mit diesen Substanzen einige orientirende Versuche angestellt, deren Resultate ich, soweit sie pharmakologisehes Interesse bieten, kurz mittheilen möchte. Zugleich führten die Versuche zu einer weiteren Untersuchung einiger Eigenschaften der Muttersubstanz der Gruppe, des Tropins.

Im Allgemeinen schien ans den bisherigen Beobachtungen hervorzugehen, dass auch die künstlichen Tropeïne wie die in der Natur vorkommenden Isomeren des Atropin qualitativ diesem gleich und nur quantitativ verschieden wirkten. Wie die folgenden Versuche aber zeigen, ergiebt die Untersuchung der neuen Tropeïne, dass sich die pharmakologische Gruppe der Tropeïne keineswegs mit der chemischen deckt, dass vielmehr bei einzelnen künstlichen Estern des Tropins die charakteristischen Wirkungen des Atropins nicht mehr nachweisbar sind, während dieselben interessante Beziehungen zu anderen Gruppen des pharmakologischen Systems erkennen lassen. Es war dabei von besonderem Interesse, die Fettsäure-Ester des Tropins kennen $\mathrm{zu}$ lernen, da solche bisher überhaupt noch nicht pharmakologisch untersucht sind. Endlich schien auch die Frage der Beachtung werth, ob die Atropinwirkungen auf Pupille und Froschherz, die nach den ähnlichen Innervationsverhältnissen beider Organe und nach der Analogie zahlreicher Giftwirkungen als zusammengehörig betrachtet werden müssen, auch bei den Veränderungen des Tropins durch Verbindung mit den verschiedenen Säureresten stets neben einander auftreten oder gleichzeitig fehlen; besonders forderte das Tropin selbst in dieser Beziehung zu neven Versuchen auf, da es nach Buch heim auf die Pupille ohne Wirkung sein, aber doch die Vagusendigungen im Sinne des Atropins lähmen sollte.

Bevor ich mich der Betrachtung der peripheren Wirkungen der Substanzen zuwende, die grösseres pharmakologisches Interesse beanspruchen dưrfen, mögen hier einige Bemerkungen über das allgemeine Vergiftungsbild Platz finden. Dasselbe ist bei den verschie- 
denen Tropeïnen sehr verschiedenartig; doch dürften wenige Notizen hierüber um so mehr genügen, da sich die Tropeïne im Thierversuche als relativ ungiftige Substanzen erwiesen. Das Tropin selbst kann bei Katzen bis $0,8 \mathrm{gr}$ pro Kilo per os injicirt werden, ohne auffallende Vergiftungserscheinungen hervorzurufen.

Im Gegensatze zu der Unwirksamkeit des Tropins auf die Pupille bei localer Einwirkung auf das Auge ist es jedoch erwähnenswerth, dass bei der Allgemeinvergiftung durch 0,2-0,5 Tropin. sulfur. als constantes Vergiftungssymptom an Katzen eine sehr auffallende Mydriasis eintritt. Die Pupillenerweiterung ist maximal und dauert Stunden lang an: die Pupille ist dabei gegen Lichteinfall reactionslos. Ueber die Ursache dieser Mydriasis habe ich keine näheren Untersuchungen angestellt; da sie auch durch wiederholte locale Application von Tropin in Substanz nicht hervorgerufen werden kann, so erweist sie sich als gänzlich verschieden von der Atropin-Mydriasis. Die Pupillenerweiterung wird ferner nicht blos durch Physostigmin prompt beeinflusst, sondern auch durch Muscarin wieder verengt.

Dem Acetyltropin kommen ausgesprochen erregende Wirkungen zu. An Fröschen stellt sich nach 0,05-0,1 Acetyltropin nur vorübergehende Narkose, nach 30-40 Minuten aber deutlich gesteigerte Reflexerregbarkeit ein. An Katzen treten nach Gaben von $0,3 \mathrm{~g}$, an Kaninchen nach $0,08-0,1 \mathrm{~g}$ heftige clonische und tonische Krämpfe ein, in denen die Thiere durch Respirationsstillstand zu Grunde gehen. Wie das Atropin selbst hat demnach auch das Acetyltropin im Vergleiche zur Muttersubstanz, Tropin erregende Eigenschaften angenommen.

Dem Succinyltropin scheinen hingegen erregende Wirkungen gänzlich abzugehen. Bei Katzen erfolgt der Tod nach Gaben über $0,5 \mathrm{~g}$ unter Lähmungserscheinungen. An Fröschen tritt schon nach Gaben von $0,02 \mathrm{~g}$. Succinyltropin. hydrobromic, nach etwa 10 bis 15 Minuten deutliche Curarewirkung auf; gleichzeitig wird aber auch das Rückenmark gelähmt.

Das Hippuryltropin zeigt an Fröschen die eigenthümliche Combination der Wirkungen der beiden ersterwähnten Tropeïne. Neben einer deutlichen Curarewirkung macht sich nämlich nach einiger Zeit eine strychninartige Wirkung auf das Rückenmark geltend, so dass nach 0,05 Hippuryltropin hydrobrom. in einer aus dem Kreislaufe ausgeschalteten Extremität nach 1-2 Stunden anf Erschütterung ein auf diese Extremität beschränkter Tetanus eintritt, während der iibrige der Curarewirkung der Substanz ausgesetzte Körper in schlaffer Lähmung verharrt. An den der Curarewirkung so viel unzugänglicheren Säugethieren tritt der Tod - an einer Katze erst nach 
$1 \mathrm{~g}$ per os - unter Erregungserscheinungen und strychninartigen Krämpfen ein.

Das Lactyltropin ist unter den untersuchten Tropeïnen das ungiftigste. Auch $1 \mathrm{~g}$ des salpetersauren Salzes wird von einer mittelgrossen Katze ohne Vergiftungssymptome vertragen.

\section{Die Wirkung der Tropeïne auf die Katzenpupille.}

Die Spaltungsproducte des Atropins besitzen bekanntlich nicht mehr seine mydriatischen Eigenschaften. Nach den übereinstimmenden Untersuchungen von Fraser ${ }^{1}$, Hell $\operatorname{mann}^{2}$ ) u. Buchheim (a.a. O.) bewirkt das Tropin selbst, als schwefelsaures Salz auch in "grösserer Menge in Substanz ins Auge gebracht" keine Pupillenerweiterung. Erst mit der Esterbildung tritt also die charakteristische Wirkung auf die Pupille auf. Dieselbe Wirkung hat nun auch, wie zuerst Bucbheim festgestellt hat, die Esterificirung mit anderen aromatischen Säuren. Von den von Laden burg dargestellten Tropeïnen wirkt insbesondere das Atrolactyltropin oder Pseudo-Atropin ganz wie Atropin and auch das Mandelsäuretropin erweitert bekanntlich die Pupille nicht weniger energiseh, wenn auch rascher vorübergehend. Auch das von Buchheim dargestellte Benzoyltropin wirkt mydriatisch; doch muss es in Substanz in Gaben von $0,001 \mathrm{~g}$ in das Auge gebracht werden, um nach einer halben Stunde beginnende Pupillenerweiterung zu bewirken.

Es musste somit von Interesse sein, ob auch die Esterificirung mit Säuren der Fettreihe dieselbe Wirkung in Erscheinung treten lässt. Bei der Essigsäure sowie bei der Bernsteinsäure ist dies nicht der Fall. Man kann diese Tropeïne in Substanz in den Conjunctivalsack von Katzen bringen, sodass eine concentrirte Lösung der leicht löslichen Substanzen einwirkt, ohne eine Beeinflussung der Pupillenweite wahrzunehmen. Die Esterbildung mit diesen Fettsäuren ist demnach für das Auftreten der mydriatischen Eigenschaften ohne Effect. Es läge der Gedanke nahe, hier einen Gegensatz in der Wirkung der Ester von aromatischen Säuren und von Fettsäuren anzunehmen. Deshalb ist es von Interesse, dass das Lactyltropin zwar schwach, aber deutlich pupillenerweiternd wirkt. 2 proc. Lösungen erzeugen allerdings noch keine Mydriasis, wenige Tropfen aber 10-20 proc. Lösung ins Auge gebracht rufen eine nach etwa einer balben Stunde beginnende Differenz in der Pupillenweite hervor.

1) Procedings of the R. Society of Edinburgh 1869 .

2) Inaug.-Diss. Jena 1873. 
Dieser Unterschied erreicht nach zwei Stunden sein Maximum; die Pupille reagirt 2 war auch dann noch träge auf Belichtung, bleibt aber etwa $3 \mathrm{mal}$ so weit als jene des gleich belichteten anderen Auges. Nach etwa 5 Stunden ist die Differenz verschwunden. Einen ganz ähnlichen Einfluss auf die Katzenpupille zeigt das Hippuryltropin.

Wie sich aber im Lactyltropin ein Fettsäureester des Tropins als mydriatisch wirksam erweist, so lassen andrerseits aromatische Ester wie Salicyltropin und Cinnamyltropin nach Falck's Versuchen 1) die mydriatische Wirkung vermissen. Es ergiebt sich demnach, dass das Tropin sowohl durch die Esterificirung mit aromatischen als auch mit Säuren der Fettreihe atropinartige Wirkung auf die Pupille erlangen kann, dass aber keineswegs allen Tropeïnen mydriatische Wirkung zukomme.

Pictet ${ }^{2}$ ) hat auf Grund der bisherigen Befunde darauf hingewiesen, dass nur jene Tropeïne mydriatisch wirkten, deren Säure ein alkoholisehes Hydroxyl enthält. Damit stimmt aber die mydriatische Wirkung des Benzoyltropins und des Hippuryltropins nicht überein und es erscheint wohl derzeit aussichtslos, nach Gesetzmässigkeiten zu suchen, in wie weit der Eintritt der Pupillenwirkung von der Constitution der esterbildenden Säure abhängig wäre.

Wirkung des Tropins und der Tropeine auf das Froschherz.

Alle untersuchten Tropeïne heben den Muscarinstiilstand des Frosehberzens auf. Ein Blick auf die folgenden Versuchsbeispiele zeigt jedoch, dass sich die Aufhebung des Muscarinstillstandes durch diese Substanzen wesentlich von der durch Atropin unterscheidet.

Versuch I (Aufhebung des Muscarinstillstandes durch Acetyltropin). Rana esculenta; Herz freigelegt.

\begin{tabular}{|c|c|c|}
\hline Zeit & $\begin{array}{l}\text { Zahl der Contrac- } \\
\text { tionen in } 1 \text { Minute }\end{array}$ & Bemerkungen \\
\hline $\begin{array}{l}10 \mathrm{~h} 42 \mathrm{~m} \\
10 \mathrm{~h} 45 \mathrm{~m}\end{array}$ & $\frac{44}{2 \text { Tropfen Muscarin }}$ in die Nähe des Her- & \\
\hline 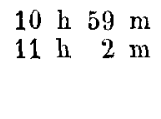 & $\begin{array}{l}\text { Stillstand complet. } \\
\text { Subcutane Inj. von } \\
0,003 \mathrm{~g} \text { Acetyltropin } \\
\text { hydrobromic. }\end{array}$ & \\
\hline $11 \mathrm{~h} 5 \mathrm{~m}$ & 1 & $\begin{array}{l}\text { Es beginnen schwache Contractionen des Ven- } \\
\text { trikels. }\end{array}$ \\
\hline $\begin{array}{llll}11 & \mathrm{~h} & 7 & \mathrm{~m} \\
11 \mathrm{~h} & 9 & \mathrm{~m}\end{array}$ & $\begin{array}{l}2 \\
4\end{array}$ & \\
\hline
\end{tabular}

1) Ladenburg, a. a. 0 .

2) Die Pflanzenalkaloide und ibre chemische Constitution. Berlin 1891. 
Ueber die Wirkungen des Tropins and der Tropeïne.

\begin{tabular}{|c|c|c|}
\hline Zeit & $\begin{array}{l}\text { Zahl der Contrac- } \\
\text { tionen in } 1 \text { Minute }\end{array}$ & Bemerkungen \\
\hline $11 \mathrm{~h} 14 \mathrm{~m}$ & 10 & Ventrikel contrahixt sich schwach. Vorhöfe ruhig. \\
\hline $11 \mathrm{~h} 20 \mathrm{~m}$ & 18 & Contraction etwas kräftiger. Diastole breit. \\
\hline $11 \mathrm{~h} 25 \mathrm{~m}$ & & Vorböfe völlig unbetheiligt. \\
\hline $\begin{array}{l}11 \mathrm{~h} 28^{1 / 2} \\
\text { bis } 30 \mathrm{~m}\end{array}$ & $\begin{array}{l}\text { Stillstand } 11 / 2 \text { Minu- } \\
\text { ten lang wieder ein- } \\
\text { getreten. }\end{array}$ & \\
\hline $11 \mathrm{~h} 31 \mathrm{~m}$ & 14 & \\
\hline $11 \mathrm{~h} 32 \mathrm{~m}$ & 20 & Ventrikelcontractionen kräftig. \\
\hline $11 \mathrm{~h} 43 \mathrm{~m}$ & 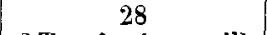 & Vorhöfe völlig ruhig. \\
\hline $11 \mathrm{~h} 44 \mathrm{~m}$ & $\begin{array}{l}2 \text { Tropfen } 3 \text { promille } \\
\text { Atropinlösung in die } \\
\text { Nähe des Herzens. }\end{array}$ & \\
\hline $11 \mathrm{~h} 46 \mathrm{~m}$ & 34 & $\begin{array}{l}\text { Herz ist, ,klein" geworden, die Contraction aus- } \\
\text { giebiger, die Entleerung des Herzens vollstän- } \\
\text { dig. Die Vorhöfe schlagen mit. }\end{array}$ \\
\hline $11 \mathrm{~h} 47 \mathrm{~m}$ & 36 & Versuch abgebrochen. \\
\hline
\end{tabular}

Versuch II (Aufhebung des Muscarinstillstandes durch Acetyltropins). Rana temporaria; Herz freigelegt.

\begin{tabular}{|c|c|c|}
\hline Zeit & $\begin{array}{l}\text { Zahl der Contrac- } \\
\text { tionen in } 1 \text { Minute }\end{array}$ & Bemerkungen \\
\hline $12 \mathrm{~h} 15 \mathrm{~m}$ & 46 & \\
\hline $12 \mathrm{~h} 16 \mathrm{~m}$ & $\begin{array}{l}2 \text { Tropfen Muscarin } \\
\text { in die Nahe des Her- } \\
\text { zens. }\end{array}$ & \\
\hline $12 \mathrm{~h} 20 \mathrm{~m}$ & Stillstand complet. & \\
\hline $12 \mathrm{~h} 25 \mathrm{~m}$ & $\begin{array}{l}\text { Subcutane Inj. von } \\
0,03 \mathrm{~g} \text { Acetyltropin } \\
\text { hydrobromic. }\end{array}$ & \\
\hline $12 \mathrm{~h} 27 \mathrm{~m}$ & 2 & Beginn schwacher Ventrikelcontractionen: \\
\hline $12 \mathrm{~h} 28 \mathrm{~m}$ & 3 & in $15 \mathrm{Sec} ;$ dann wieder Stillstand bis \\
\hline $12 \mathrm{~h} 29 \mathrm{~m}$ & 3 & in $15-20$ Sec, dann folgt wieder Stillstand \\
\hline $12 \mathrm{~h} 30 \mathrm{~m}$ & 5 & in 30 Sec., dann Stillstand von etwa $1 \mathrm{~m}$. \\
\hline $12 \mathrm{~h} 32 \mathrm{~m}$ & 2 & \\
\hline $12 \mathrm{~h} 34 \mathrm{~m}$ & 4 & \\
\hline $12 \mathrm{~h} 36 \mathrm{~m}$ & 19 & Herzcontractionen sind rhythmisch. \\
\hline $12 \mathrm{~h} 40 \mathrm{~m}$ & 22 & $\begin{array}{l}\text { Ventrikelcontractionen ziemlich kräftig, die Vor- } \\
\text { höfe noch ruhig. }\end{array}$ \\
\hline $12 \mathrm{~h} 45 \mathrm{~m}$ & 24 & Auch die Vorböfe contrahiren sich. \\
\hline & 24 & $\begin{array}{l}\text { Kraftige Systole, aber Diastole von Muscarin- } \\
\text { charakter. }\end{array}$ \\
\hline $\begin{array}{l}12 \mathrm{~h} 52 \mathrm{~m} \\
12 \mathrm{~h} 55 \mathrm{~m}\end{array}$ & $\begin{array}{c}\text { Subcutane Inj. von } \\
0,003 \text { Atropin. } \\
40\end{array}$ & Sehr kräftige Systole und normale Diastole. \\
\hline
\end{tabular}

Ganz wie das Essigsäure-Tropin verhalten sich Bernsteinsäureund Hippursäure-Tropin. Es sind zur Aufhebung des Muscarinstillstandes im Vergleich zum Atropin sehr hohe Gaben nothwendig (etwa die 1000 fache Gabe des Atropin). Aber anch abgesehen von diesem rein quantitativen Unterschiede machen die Erscheinungen bei der Aufhebung einen wesentlich anderen Eindruck. Denn während 
auch nach kleinsten Atropingaben (z. B. 1/200 mg) den ersten Contractionen des Herzens bald schnellere Pulse folgen und die Herzthätigkeit nach einiger Zeit ihren normalen Typus wiedererhält wird durch die genannten Tropeïne die Hemmung niemals vollständig überwunden, der Charakter der Muscarinwirkung bleibt vielmehr auch nach Aufhebung des Stillstandes durch die "Grösse" des Herzens, seine ungenügende Entleerung und breite Diastole ausgeprägt. Die Schlagfolge des Herzens erreicht auch nicht ihren normalen Werth und eine kleine nachfolgende Atropingabe ruft stets noch Beschleunigung hervor. Fast immer erfolgt die Aufhebung des diastolischen Stillstandes sehr allmählich und häufig kann man dabei eine eigenthümliche "Gruppenbildung" des Pulses beobachten, indem Anfangs 3-5 Ventrikelcontractionen innerhalb $1 / 4-1 / 2$ Minute erfolgen, zwischen diese Gruppen sich aber wieder diastolische Stillstände von $1 / 2$ bis 1 Minute Dauer zwischenschieben (s. Versuch II); oft tritt auch nach minutenlangem, ziemlich frequentem Schlagen des Vertikels unvermittelt ein länger dauernder diastolischer Stillstand wieder ein. Die Vorhöfe betheiligen sich an den Pulsen stets erst nach längerer Zeit, ja oft erst, wenn der Ventrikel bereits 20-25 Contractionen in der Minute ausfiuhrt. Vor Allem aber muss betont werden, dass auch nach Mitbetheiligung der Vorhöfe die Herzthätigkeit das normale Bild nicht wieder gewinnt, das nach gewisser Zeit auch kleinste Atropingaben herbeifithren.

Das Lactyltropin weicht von dem geschilderten Verhalten insofern ab, als nach grossen Gaben von 2-5 cg die Erscheinungen der Aufhebung mehr denen nach Atropin gleichen und die Aufhebung eine vollständige werden kann. Bei den anderen Tropeïnen wurde Aehnliches nur bei Hippuryltropin beobachtet, wenn dasselbe in 2 proc. Lösung in die. Nähe des Herzens geträufelt wurde, nicht aber nach subcutaner Injection. Kleinere Gaben Lactyltropin verhalten sich wie Acetyltropin.

Versuch III (Aufhebung des Muscarinstillstandes durch Lactyltropin). Rana temporaria; Herz freigelegt.

\begin{tabular}{|c|c|c|}
\hline Zeit & $\begin{array}{l}\text { Herzcontractionen } \\
\text { in } 1 \text { Minute }\end{array}$ & Bemerkungen \\
\hline $\begin{array}{llll}11 & \mathrm{~h} & 30 & \mathrm{~m} \\
11 & \mathrm{~h} & 32 & \mathrm{~m} \\
& & & \\
11 & \mathrm{~h} & 40 & \mathrm{~m} \\
11 & \mathrm{~h} & 45 & \mathrm{~m}\end{array}$ & $\mid \begin{array}{c}50 \\
2 \text { Tropf. Muscarin in } \\
\text { die Nähe der Herzens. } \\
\text { Stillstand complet. } \\
\text { Subcutane Inj. von } \\
0,005 \text { g Lactyltropin } \\
\text { nitric. }\end{array}$ & \\
\hline
\end{tabular}


Ueber die Wirkungen des Tropins und der Tropeïne.

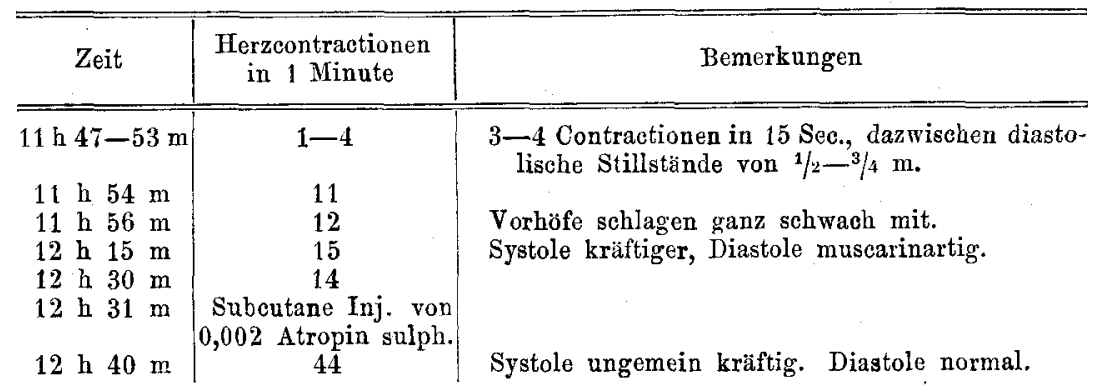

Versuch IV. (Aufhebung des Muscarinstillstandes durch Lactyltropin in grosser Gabe).

\begin{tabular}{|c|c|c|}
\hline Zeit & $\begin{array}{l}\text { Herzcontractionen } \\
\text { in } 1 \text { Minute }\end{array}$ & Bemerkungen \\
\hline $\begin{array}{llll}12 & \mathrm{~h} & 25 & \mathrm{~m} \\
12 & \mathrm{~h} & 28 & \mathrm{~m}\end{array}$ & $\begin{array}{l}\text { Ho } \\
\text { Herz durch } 1 \text { Tropf. } \\
\text { Muscarin zum Still- } \\
\text { stand gebrackt. }\end{array}$ & \\
\hline $12 \mathrm{~h} 32 \mathrm{~m}$ & $\begin{array}{l}\text { Subeutane Injection } \\
\text { von } 0,02 \text { Lactyltropin } \\
\text { nitric. }\end{array}$ & \\
\hline $12 \mathrm{~h} 33 \mathrm{~m}$ & 4 & \\
\hline $12 \mathrm{~h} 34 \mathrm{~m}$ & 26 & Vorhöfe schlagen schwach mit. \\
\hline $12 \mathrm{~h} 35 \mathrm{~m}$ & 29 & \\
\hline $12 \mathrm{~h} 40 \mathrm{~m}$ & 26 & Krüftige Pulse. \\
\hline 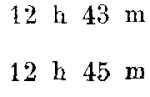 & \begin{tabular}{|}
3 Tropfen 1 pro mille \\
Atropin auf das Herz. \\
32
\end{tabular} & Versuch abgebrochen. \\
\hline
\end{tabular}

Mit der geschilderten Unvollständigkeit der Aufhebung steht die Thatsache im Einklang, dass auch nach grossen Gaben dieser Tropeïne die Vagusreizung nicht vollkommen unwirksam wird. Ebenso bleibt auch nach längerer Einwirkung sehr grosser Gaben das Muscarin wirksam und vermag bedeutende Verlangsamung der Schlagfolge mit lang andauernden Diastolen, wenn auch nicht mehr Stillstand herbeizufïhren.

Versuch V (Wirkung des Muscarin nach Acetyltropin). Rana esculenta; Herz freigelegt.

\begin{tabular}{|c|c|c|}
\hline Zeit & $\mid \begin{array}{c}\text { Zahl der Herzeon- } \\
\text { tractionen in } 1 \text { Min. }\end{array}$ & Bemerkungen \\
\hline $\begin{array}{llll}11 & \mathrm{~h} & 21 & \mathrm{~m} \\
11 & \mathrm{~h} & 22 & \mathrm{~m}\end{array}$ & $\begin{array}{l}46 \\
\text { Subcutane Inj. von } \\
0,05 \text { Acetyltropin. }\end{array}$ & \\
\hline $\begin{array}{llll}11 & \mathrm{~h} & 24 & \mathrm{~m} \\
11 & \mathrm{~h} & 28 & \mathrm{~m} \\
11 & \mathrm{~h} & 35 & \mathrm{~m}\end{array}$ & $\begin{array}{l}48 \\
46 \\
42\end{array}$ & $\begin{array}{l}\text { Systole deutlich verlängert, Diastole sehr kurz. } \\
\text { Systole lang. Herzspitze bleibt auch in der Dia- } \\
\text { stole contrahirt. }\end{array}$ \\
\hline
\end{tabular}




\begin{tabular}{|c|c|c|}
\hline Zeit & $\begin{array}{l}\text { Zahl der Herzeon- } \\
\text { tractionen in } 1 \text { Min. }\end{array}$ & Bemerkungen \\
\hline $\begin{array}{llll}11 & \mathrm{~h} & 45 & \mathrm{~m} \\
11 & \mathrm{~h} & 46 & \mathrm{~m}\end{array}$ & $\begin{array}{l}42 \\
2 \text { Tropfen Muscarin } \\
\text { auf das Herz. }\end{array}$ & \\
\hline $11 \mathrm{~h} 47 \mathrm{~m}$ & 34 & Diastole wird breit. \\
\hline $11 \mathrm{~h} 53 \mathrm{~m}$ & 26 & Systole sehr kräftig, Diastole muscarinartig. \\
\hline $12 \mathrm{~h}-\mathrm{m}$ & 12 & Vorböfe bleiben in diastolischem stillstand. \\
\hline $12 \mathrm{~h} 15 \mathrm{~m}$ & 8 & $\begin{array}{l}\text { Completer Stillstand tritt auch in der Folge } \\
\text { nioht ein. }\end{array}$ \\
\hline
\end{tabular}

Versuch VI (Wirkung von Muscarin nach Lactyltropin). Rana temporaria; Herz freigelegt.

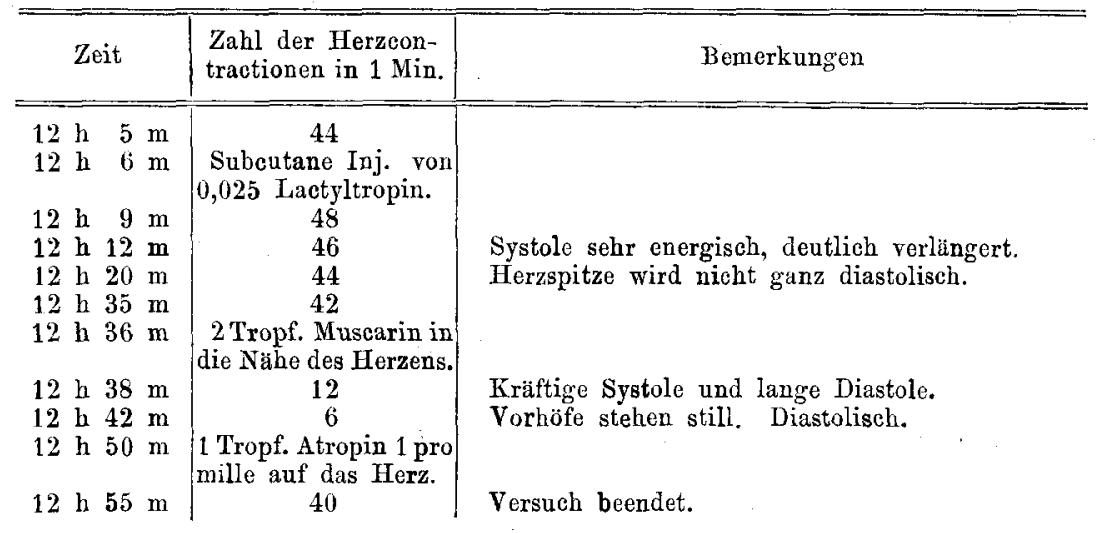

Es entsteht die Frage, welche Deutung den geschilderten Erscheinungen zu geben ist; ob die genannten Tropeine de m Atropin analog und nur quantitativ von ihm versehieden auf das Froschherz einwirken oder ob sie sich vielmehr einer Reihe anderer Substanzen anschliessen, die den Muscarinstillstand in indirecter Weise beeinflussen. In der That gleicht die Aufbebung des Stillstandes weit mehr der Wirkung von Physostigmin oder Campher auf das muscarinisirte Froschberz, als jenen des Atropins und das Verhalten der Tropeïne dem Muscarin und Vagusreize gegenïber liesse sich auch durch die Annahme erklären, dass ihre Wirkung nicht die Hemmungsapparate selbst, sondern andere nervöse Elemente im Herzen oder den Herzmuskel selbst betrifft. Um der Frage näher zu treten, musste untersucht werden, ob sich in der Beeinflussung des nicht muscarinisirten Froschherzens Anhaltspunkte für eine solche, auf andere als die Hemmungsapparate im Herzen gerichtete Wirkung ergeben.

Die untersuchten Tropeïne rufen in Gaben von $1-2 \mathrm{eg}$ nach einer inconstanten vorubbergehenden Beschleunigung des Herzschlages 
eine Verlangsamung der Herzaction hervor; neben dieser geringen Abnahme der Pulszahl macht sich aber eine deutliche Verlängerung und Verstärkung der Systole geltend, die insbesondere bei Acetyl- und Lactyltropin sehr ausgesprochen ist (vgl. Versuch V u. VI). Insbesondere an Rana temporaria bemerkt man, dass die Contraction energischer wird und der Ventrikel länger in Systole verharrt, als vor der Einwirkung des Giftes. Die Wirkung bleibt lange Zeit bestehen und nur nach sehr grossen Gaben wird die Anfangs energische Contraction in der Folge allmählich kraftloser. Vorübergehend tritt ferner nach grösseren Gaben ein der sogenannten Herzperistaltik ähnlicher Zustand ein, indem einzelne Theile des Ventrikels in der Systole sich nicht entleeren und als Höcker vorgewölbt bleiben, während die Herzspitze in der Diastole etwas contrahirt bleibt. Niemals aber kommt es zu einem systolischen Stillstand, vielmebr wird die lang danernde Systole allmählich unvollständiger und nach stundenlanger Beobachtung sieht man das Herz in Lähmung übergehen.

Die geschilderte Veränderung der Herzthätigkeit kann am einfachsten durch eine Füblhebelvorrichtung, wie sie Ka is e ${ }^{1}$ ) beschrieben hat, graphisch dargestellt und die Verlängerung der systolischen Phase an der Curve gemessen werden. Ein derartiger Fublhebel schreibt, auf die Ventrikelbasis aufgesetzt, ohne die Herzthätigkeit wesentlich zu verändern, eine Curve, die von $\mathrm{K}$ aiser ausführlich discutirt ist und an der sich Beginn und Ende der einzelnen Herzphasen deutlich ausprägen, so dass durch Ordinaten der betreffenden Curvenpunkte bei gleichzeitiger Registrirung von 1/s Secunden mittelst des Jaquet'schen Chronoskops die Dauer von Systole und Diastole in bequemer Weise gemessen werden kann. Die beigegebenen 3 Figuren zeigen Curvenabschnitte aus dem folgenden Versuche; in I sind die normalen Herzbewegungen registrirt, in II ihre Veränderung 15 Minuten nach Injection von Acetyltropin, in III 30 Minuten nach derselben. In ähnlicher Weise lässt sich die Veränderung der Herzthätigkeit mittelst der Engel mann'schen Suspensionsmethode registriren und Herr Cand. med. Schiller wird über solche Versuche nächstens berichten.

Versuch VII (Wirkung von Acetyltropin auf die einzelnen Phasen del Herzthätigkeit). Rana temporaria schwach curarisirt; Herz freigelegt und mit dem Kaiser'schen Fühlhebel armirt.

\begin{tabular}{c|c|cc}
\hline Zeit & $\begin{array}{c}\text { Zahl der Horzcon- } \\
\text { tractionen pro 1 Min. }\end{array}$ & Dauer von Systole und Dastole in 1/10 See. \\
\hline $10 \mathrm{~h} 15 \mathrm{~m}$ & 50 & Systole 6 & Diastole 5. \\
$10 \mathrm{~h} 20 \mathrm{~m}$ & 49 & Systole 6 & Diastole 5.
\end{tabular}

1) Zeitschr. f. Biologie. 1593. S. 203. 


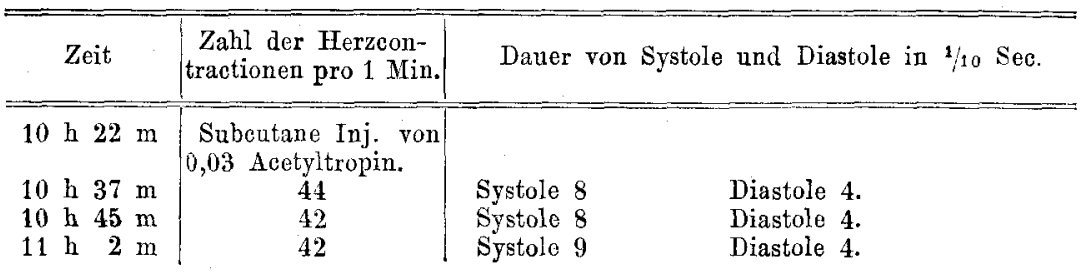

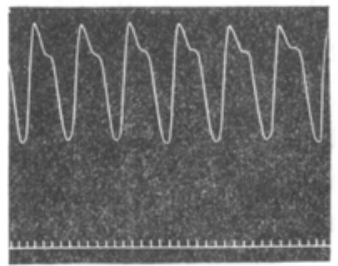

Curve 1. (Normal.)

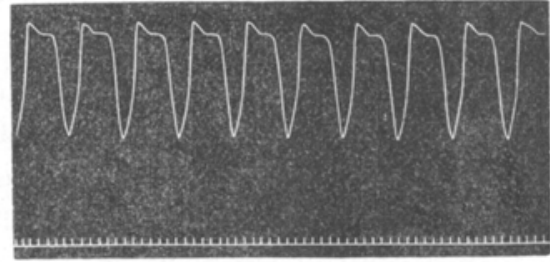

Curve 2. 15 Nin. nach Acetyltropin.

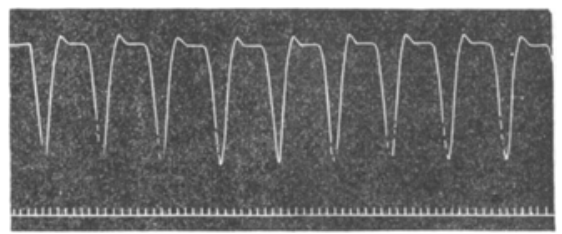

Curve 3. 30 Min. nach Acetyltropin.

Anch am Williams'schen Frosebherzapparate ist die Verlängerung der Systole kenntlich. Hier spricht sich aber mit der Verlangsamung der Pulse auch die Verstärkung der einzelnen Contractionen durch ein Höherwerden der Pulse und eine entsprechende Vergrösserung des Pulsvolums, sowie durch geringes Ansteigen des mittleren Druckes aus. Die absolute Kraft des Herzens bleibt dabei unverändert.

Versuch VIII (Wirkung von Lactyltropin am Williams'schen Apparate).

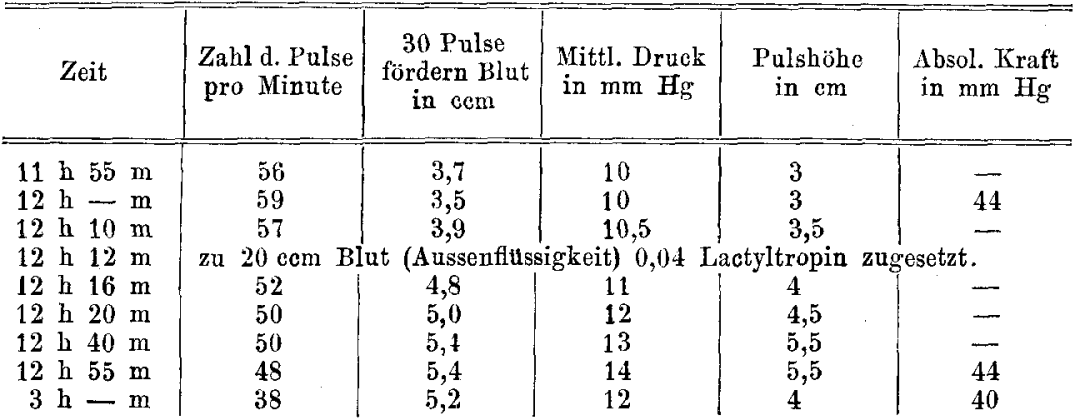

Die Tropeine, insbesondere Acetyl- und Lactytropin beeinflussen demnach die Thätigkeit des Froschberzens durch Verlängerung und 
Verstärkung der systolischen Contraction, sie steigern die Neigung des Ventrikels in Systole zu verbarren. Diese Wirkung erinnert am meisten an das erste Stadium der Digitalinwirkung, für welches dieses Symptom besonders von Dreser ${ }^{1}$ ) hervorgehoben wurde. Am besten lässt sich die Veränderung in dem Verhältniss der einzelnen Herzphasen beobachten und mittelst des Fühlhebels oder der Engelmann'sehen Suspensionsmethode registriren nach kleinsten Helleboreïngaben, nach denen der systolische Stillstand erst nach längerer Zeit $(1 / 2-1$ Stunde) eintritt.

Kebren wir nun zu dem Ausgangspunkte dieser Untersuchung zurück, so muss die Frage aufgeworfen werden, ob die geschilderte Beeinflussung der Herzthätigkeit geeignet erscheint, die Aufhebung des Muscarinstillstands durch die Substanzen zu erklären? Schon $\mathrm{B} \ddot{o} \mathrm{~h} \mathrm{~m}^{2}$ ) hat die Angabe gemacht, dass Digitalin den Muscarinstillstand aufhebt. In welcher Weise aber jene kleinsten Helleboreïngaben, die erst nach längerer Zeit systolischen Stillstand herbeiführen, den Muscarinstillstand beeinflassen, mag das folgende Versuchsbeispiel illustriren.

Versuch IX (Aufhebung des Muscarinstillstandes durch 0,0005 Helleboreïn). Rana esculenta; Herz freigelegt.

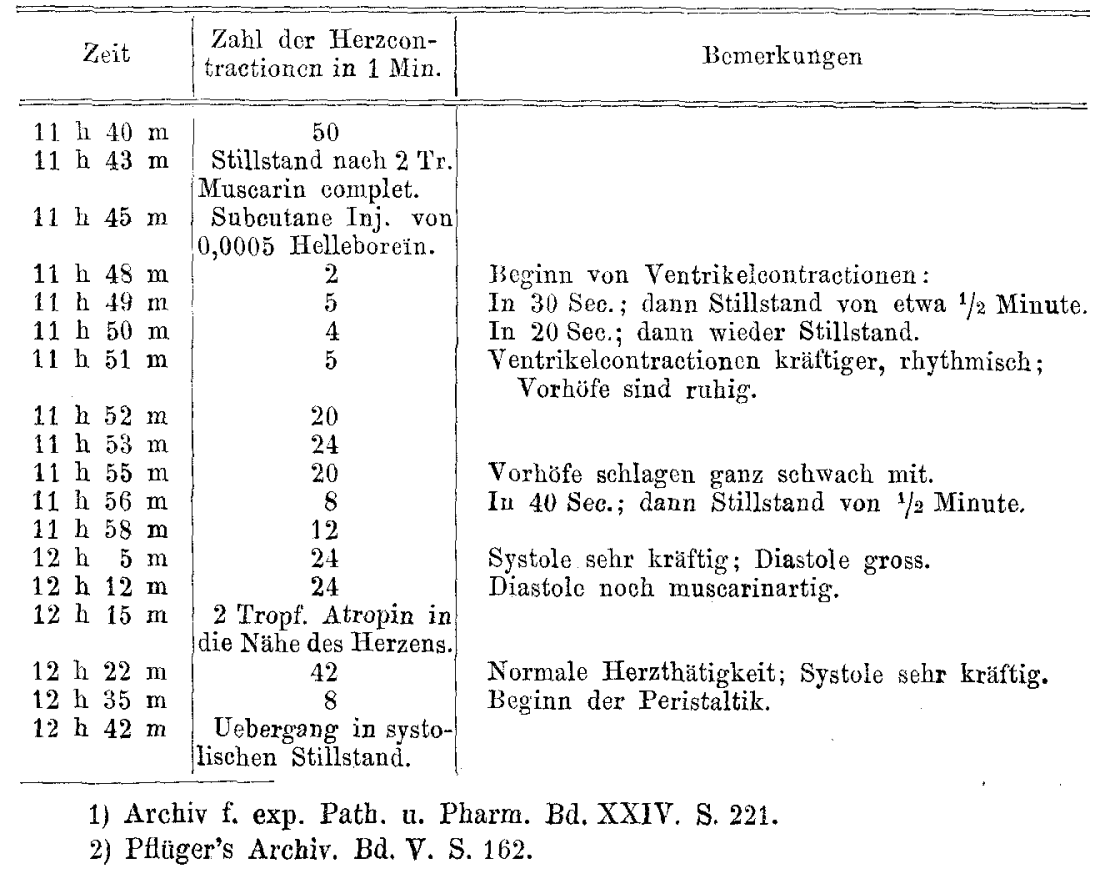


Der Versuch zeigt, dass auch kleinste Helleboreïngaben den Muscarinstillstand aufheben und ziemlich frequente, kräftige Ventrikelcontractionen anregen, bevor das Herz durch die typische Digitaliswirkung in systolischen Stillstand übergeht: Die Aufhebung ist aber eine unvollständige, der Charakter der Muscarinwirkung spricht sich auch nach der Aufhebung in der Schlagfolge des Herzens aus und erst eine geringe Gabe Atropin führt zu normaler Frequenz und normalem Typus der Herzthätigkeit. Die Aufhebung des Muscarinstillstandes durch kleinste Helleboreïngaben bietet somit die grösste Aebnlichkeit mit jener durch Tropeine, wenn auch der weitere Verlauf der Vergiftung durch den systolischen Stillstand nach Helleboreïn einen so durchgreifenden Unterschied zeigt. Auch diese Auf hebung ist charakterisirt durch ihre Unvollständigkeit; ja auch jenes eigenthïmliche Symptom der "Gruppenbildung" der Ventrikelpulse und die diastolischen Stillstände, die häufig die Aufhebung unterbrechen, findet sich nach Helleborein wieder. Andrerseits hat $\mathrm{Kais}$ er ${ }^{1}$ ) gezeigt, dass auch nach grösseren Helleboreïngaben und unmittelbar vor dem systolischen Stillstande Muscarin und Vagusreiz ihre Wirksamkeit nicht eingebüsst haben.

Erinnert somit die Wirkung einzelner Tropeïne auf das Froschherz an das erste Stadium der Digitaliswirkung, so heben andrerseits kleinste Helleboreïngaben in diesem ersten Stadium den Muscarinstillstand in sehr ähnlicher Weise auf und es liegt der Schluss nabe, dass es sich auch bei den Tropeïnen nicht um eine atropinartige Lähmung der Hemmungsapparate, sondern um Beeinflussung anderer Theile des Herzens handelt. Freilich die Annahme, dass neben der nachweisbaren Reizwirkung auf das Herz ihnen noch eine atropinartige Wirkung zukomme, die nur qualitativ so gering ist, dass sie sich am nicht-muscarinisirten Herzen nicht nachweisen liesse, kann nicht mit Sicherheit ausgeschlossen werden. Doch genügt die nachgewiesene Reizwirkung auf das Herz zur Erklärung aller Erscheinungen.

Die Annahme einer nur quantitativ weit geringeren Atropinwirkung wird vor Allem durch die Abstammung der Substanzen von dem Tropin nahegelegt, dem schon selbst die Wirkung auf die Vagusenden zukommen sollte. Es musste deshalb auf die Wirkung der Muttersubstanz näher eingegangen werden.

Wie schon erwähnt, wirkt das Tropin auch in Substanz in's Auge gebracht, nicht mydriatisch. Nach den älteren Untersuchungen Buchbeims, Hellmanns u. A. sollte es aber auf die Vagusenden im Sinne des Atropins, wenn auch weit schwächer einwirken. In

1) Zeitschr. f. Biologie. Bd. XXX. S. 393. 
diesem Verbalten läge ein auffallender Gegensatz; denn die Uebereinstimmung in den Innervationsverhältnissen der Pupille und des Froschberzens ist eine so weitgehende und bedingt den Parallelismus der Wirkung auf die beiden Organe in einer so zahlreichen Reihe von Giften, dass das isolirte Auftreten der einen Wirkung ohne die andere befremden muss. Mit Riicksicht auf die Beobachtungen an den Tropeïnen musste deshalb die Frage nach der Wirkung des Tropins anf das muscarinisirte Froschberz neu aufgenommen werden.

Versuch X (Aufhebung des Muscarinstillstandes durch Tropin). Rana esculenta; Herz freigelegt.

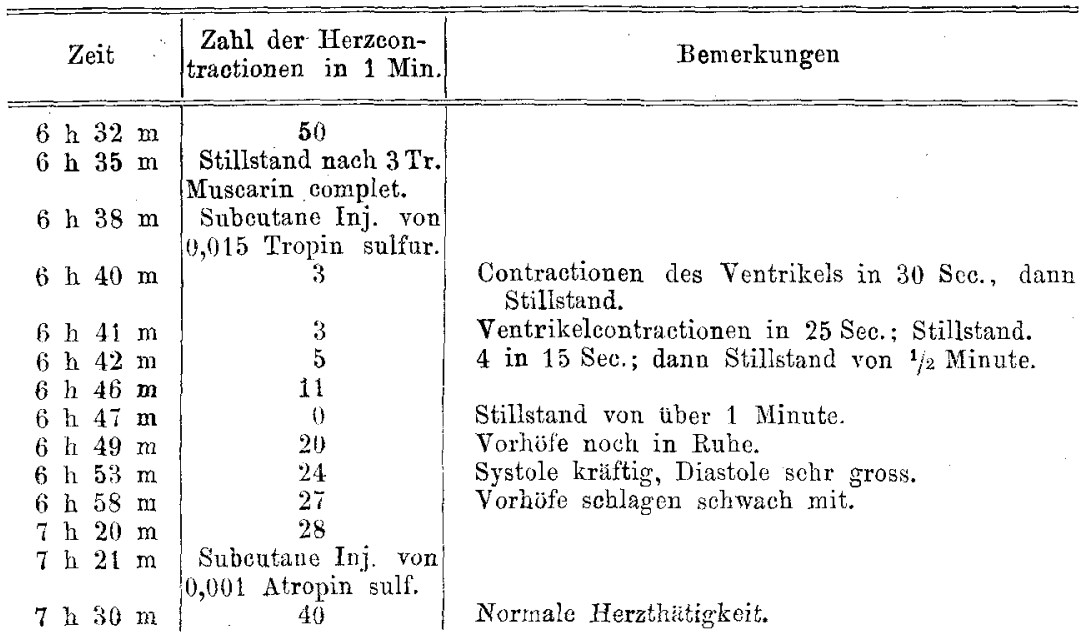

Versuch XI. (Wirkung von Muscarin nach Tropin). Rana temporaria; Herz freigelegt.

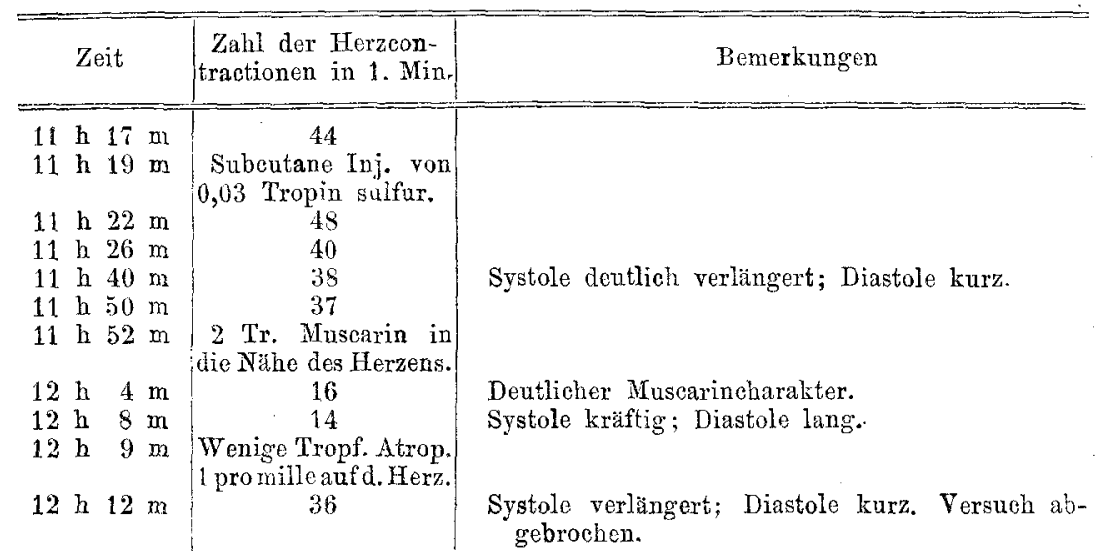


Die Versuche zeigen, dass die Aufhebung des Muscarinstillstandes durch Tropin von der durch kleinste Atropingaben versehieden und in sehr ähnlicher Weise erfolgt, wie etwa durch Acetyltropin. Es sind im Vergleiche zum Atropin sehr grosse Gaben $(3-5 \mathrm{mg})$ dazu nothwendig, die Aufhebung erfolgt allmählich unter "Gruppenbildung" der Ventrikelcontractionen und bleibt eine unvollständige; öfters tritt nach erfolgter Aufhebung vorïbergehend wieder diastolischer Stillstand ein und erst eine nachträgliche Atropingabe stellt den normalen Typus der Herzthätigkeit wieder her. Hingegen wird auch nach sehr grossen Tropingaben Muscarin nicht ganz unwirksam. Da Tropin ferner in ähnlicher Weise, - wenn auch vielleicht nicht so ausgeprägt, - wie z. B. Acetyltropin die systolische Phase des Herzens verlängert und verstärkt, sowie am Williams'schen Apparate eine Erlı̈hung der Pulse und Vergrösserung des Pulsvolums hervorruft, so wird man es als Reizmittel für das Herz ansprechen müssen und annehmen dürfen, dass auch Tropin nicht auf die Vagusendigungen wirkt, sondern durch einen Reiz auf andere Elemente des Herzens den Musearinstillstand a ufhebt.

Durch diese Deutung der Tropinwirkung wird es verständlich, dass zwar alle untersuchten Tropeïne den Muscarinstillstand beeinflussen, aber nur bei wenigen der Ester eine Lähmung der Hemmungsapparate nachweisbar ist; die Reizwirkung auf das Herz käme eben der ganzen Gruppe za, die Atropinwirkung aber nur einzelnen Abkömmlingen des Tropin. Es verschwindet ferner durch die gegebene Deutung der Erscheinungen der erwähnte Gegensatz zwischen der Unwirksamkeit des Tropin auf die Pupille und der Herzwirkung der Substanz.

Dass dem Tropin und seinen Estern eine stimulirende Wirkung. auf das Froschberz zukommt, lässt sich am besten unter pathologischen Verhältnissen erweisen. Das unter dem Einfluss einer muskellähmenden Substanz, z. B. des Kupfers langsam und kraftlos schlagende Froschherz wird so durch Tropin oder Acetyl- und Lactyltropin zu kräftigeren Systolen angeregt. Am Williams'schen Froschherzapparate kann in ähnlichen Versuchen, wie sie Barnack mit Phy. sostigmin angestellt hat, erwiesen werden, dass der durch Kupfer hervorgerufene Stillstand des Froschberzens durch Tropin für kurze Zeit aufgehoben wird. Setzt man nämlich zur Blutkochsalzmischung, die das Froschherz durchströmt, so lange kleine Gaben von weinsaurem Kupferoxydnatron hinzu, bis die Herzthätigkeit kein Blnt 
mehr in den künstlichen Kreislauf fördert, keine Pulse mehr geschrieben werden und auch an dem Plethysmographen, in welchem das Herz arbeitet, nur mebr Ausschläge von wenigen Zehntel cm wahrgenommen werden, bis das Herz also fast völlig stillsteht, und lässt nun rasch Blutmischung mit einem Zusatz von 0,02 Tropin hindurchfliessen, so setzt das Herz nach wenigen Minuten mit neuen Pulsen ein, es wird wieder Blut in den künstlichen Kreislauf gefördert, Pulse geschrieben und sogar recht kräftige Systolen ausgeführt, bis das Herz durch die Muskelwirkung des Kupfers mechaniseh nnerregbar wird und endgiltig zum Stillstand kommt.

Versuch XII (Kupfer und Tropin am Williams'schen Apparate).

\begin{tabular}{|c|c|c|c|c|c|}
\hline Zeit & 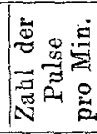 & $\begin{array}{l}\text { Pulsvolumen } \\
\text { (plethys- } \\
\text { mographisch) } \\
\text { in } \mathrm{cm}\end{array}$ & 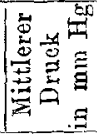 & 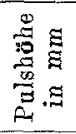 & Bemerkungen \\
\hline $5 \mathrm{~h} 30 \mathrm{~m}$ & 38 & 2,6 & 12,5 & 4,0 & \\
\hline $5 \mathrm{~h} 32 \mathrm{~m}$ & - & - & - & $\ldots$ & $\begin{array}{l}\text { Weinsaures Kupferoxydnatron dem In- } \\
\text { nenblut zugesetzt ( } 3 \mathrm{mg} \text { CuO). }\end{array}$ \\
\hline $5 \mathrm{~b} 35 \mathrm{~m}$ & 26 & 2,7 & 9,5 & 4,0 & \\
\hline $5 \mathrm{~h} 39 \mathrm{~m}$ & 20) & 2,4 & 8,0 & - & Peristaltik. \\
\hline $5 \mathrm{~h} 48 \mathrm{~m}$ & 20 & 2,6 & 9,0 & 3,5 & Wieder rogelmässig. \\
\hline $5 \mathrm{~h} 50 \mathrm{~m}$ & - & - & - & - & $\begin{array}{l}\text { Nochmals Kupferoxydnatron (3 } \mathrm{mg} \\
\text { CuO). }\end{array}$ \\
\hline $5 \mathrm{~h} 52 \mathrm{~m}$ & 12 & 1,5 & 7,0 & 2,0 & \\
\hline $5 \mathrm{~h} 53 \mathrm{~m}$ & - & $0,5-0,6$ & 5,0 & 0 & \\
\hline $5 \mathrm{~h} 53 \mathrm{~m}$ & - & $\ldots$ & $\ldots$ & - & 0,03 Tropin zur Innenflüssigkeit. \\
\hline $5 \mathrm{~h} 56 \mathrm{~m}$ & 16 & $1,6-1,8$ & 8,0 & 2,5 & \\
\hline $6 \mathrm{~h}-\mathrm{m}$ & 15 & $1,8-2,0$ & 8,0 & 2,0 & \\
\hline $6 \mathrm{~h} 3 \mathrm{~m}$ & 15 & 1,2 & 6,5 & 1,5 & \\
\hline $6 \mathrm{~h} 5 \mathrm{~m}$ & - & $0,3-0,4$ & 5,3 & 1,0 & \\
\hline $6 \mathrm{~h} 8 \mathrm{~m}$ & - & & - & $\cdots$ & $\begin{array}{l}\text { Stillstand complet. Herz mechanisch } \\
\text { unerregbar. }\end{array}$ \\
\hline
\end{tabular}

Wir haben uns nun der Frage zuzuwenden, a $f$ welche Apparate im Herzen die erregende Wirkung des Tropins und der Tropeïne gerichtet ist, ob auf nervöse Elemente oder auf den Herzmuskel selbst. Die Pharmakologie wird ja gewiss bis auf weiteres an der älteren Lehre von der motorischen Natur der intracardialen Ganglien festzuhalten haben, da bisher keine Möglichkeit angedeutet erscheint von dem Boden einer rein musculären Theorie der Herzbewegung aus die so ungemein complicirten Erscheinungen der Herzpharmakologie dem Verständniss näher zu bringen. Bei den Tropeïnen erscheint von vornherein die Beeinflussung nervöser Elemente wahrscheinlicher, da sich bei ihnen im Gegensatze, z. B. zu dem Pbysostigmin eine Wirkung auf den Skeletmuskel nicht nachweisen lässt. Auch das beschriebene Symptom der "Gruppenbildung“6 
der Pulse bei der Aufhebung des Muscarinstillstands scheint mehr für eine nervöse Wirkung zu sprechen. Weitere Anbaltspunkte dafür ergeben sich aber aus dem antagonistischen Verbalten der Substanzen gegenüber muskelläbmenden und andererseits gegenüber unzweifelhaft ganglienlähmenden Giften. In der Beeinflussung dieser pathologischen Zustände am Herzen macht sich nämlich ein Unterschied geltend, durch welchen gestiitzt auf Harna $c \mathrm{k}$ 's Untersuchungen auf diesem Gebiete eine nähere Analyse möglich wird.

Das nach Injection eines muskellähmenden Giftes, z. B. einer neutralen Kupferlösung langsam und kraftlos schlagende Herz contrahirt sich nach einigen Centigrammen Tropin energischer. Demgemäss wird in dem angefïhrten Versuche am Williams'schen Apparate anch der Kupferstillstand des Herzens durch Tropin oder Tropeïne behoben. Bei herabgesetzter Erregbarkeit des Herzmuskels behalten also die Substanzen ihre charakteristische Eigenschaft die Systole zu verstärken bei. Anders aber, wenn die Erregbarkeit der motorischen Herzganglien durch das Gift herabgesetzt ist! Sind, wie bei Chloralhydrat, die Ganglien der erste Angriffspunkt des Giftes, so bleiben die Tropeïne ohne Wirkung auf Frequenz und Stärke des Herzschlags und es erfolgt Stillstand, obgleich der Ventrikel meehanisch noch sebr gut erregbar sein kann.

Versuch XIII (Chloralhydrat und Acetyltropin). Rana temporaria; Herz freigelegt.

\begin{tabular}{|c|c|c|}
\hline Zeit & $\begin{array}{l}\text { Zahl der Herz- } \\
\text { contractionen }\end{array}$ & Bemerkungen \\
\hline $\begin{array}{lll}11 & \mathrm{~b} & 20 \mathrm{~m} \\
11 & \mathrm{~h} & 22 \mathrm{~m}\end{array}$ & $\begin{array}{l}\text { 0,08 Chloralbydrat } \\
\text { subcutan. }\end{array}$ & \\
\hline $\begin{array}{llll}11 & \mathrm{~h} & 40 \mathrm{~m} \\
11 & \mathrm{~h} & 45 \mathrm{~m} \\
11 \mathrm{~h} & 46 \mathrm{~m}\end{array}$ & $\begin{array}{c}10 \\
8 \\
\text { Subcutane Inj. von } \\
0,01 \text { Acetyltropin. }\end{array}$ & $\begin{array}{l}\text { Systole kräftig; lange diastolische Pause. } \\
\text { Vorhöfe arbeiten kräftig, Ventrikel schlechter. }\end{array}$ \\
\hline $\begin{array}{l}11 \mathrm{~h} 50 \mathrm{~m} \\
12 \mathrm{~h}-\mathrm{m}\end{array}$ & $\begin{array}{c}6 \\
\text { Diastolischer Stillstan } \\
\text { chanisch reizbar. }\end{array}$ & des Herzens, obgleich der Ventrikel noch me- \\
\hline
\end{tabular}

Wenn die Erregbarkeit der motorisehen Herzganglien unter ein gewisses Niveau gesunken ist, so bleibt Tropin demnach ohne Wirkung, auch wenn die Erregbarkeit des Herzmuskels noch erhalten ist. Substanzen aber, welche einen Reiz auf den Herzmuskel ausüben, sind unter den gleichen Verhältnissen im Stande den diastolischen Stillstand mit Sicherheit aufaubeben, wie es Harnack und Witkowski ${ }^{1}$ ) für das Physostigmin dargethan haben. Es wird da-

1) Archiv f. exp. Path. u. Pharm. Bd. V. S. 401. 
durch wahrscheinlich, dass die erregende Wirkung der Tropeïne an denselben Apparaten angreift, welche durch Chloralhydrat gelähmt werden, an den motorischen Herzganglien.

Dafür sprechen ferner Versuche, die ich nach der von Harnack in seinen bekannten Physostigminversuchen zuerst angewandten Anordnung anstellte. Hat man nämlich den Muscarinstillstand durch Tropin oder Tropeîne aufgeboben, so kann man ihn durch Injection einer genügenden Gabe Chloralhydrat wieder eintreten sehen; der Herzmuskel bleibt dabei erregbar und Atropin ruft neuerdings Pulse hervor. Niemals aber konnte ich den Wiedereintritt des Muscarinstillstandes nach Injection mauskellähmender Gifte, z. B. durch Kupfer beobachten; vielmehr werden hierdurch nur die Herzbewegungen kraftloser, bis endlich nach längerer Zeit Stillstand in Mittelstellung eintritt.

Versuch XIV (Muscarin-Tropin-Chloralhydrat). Rana temporaria; Herz freigelegt.

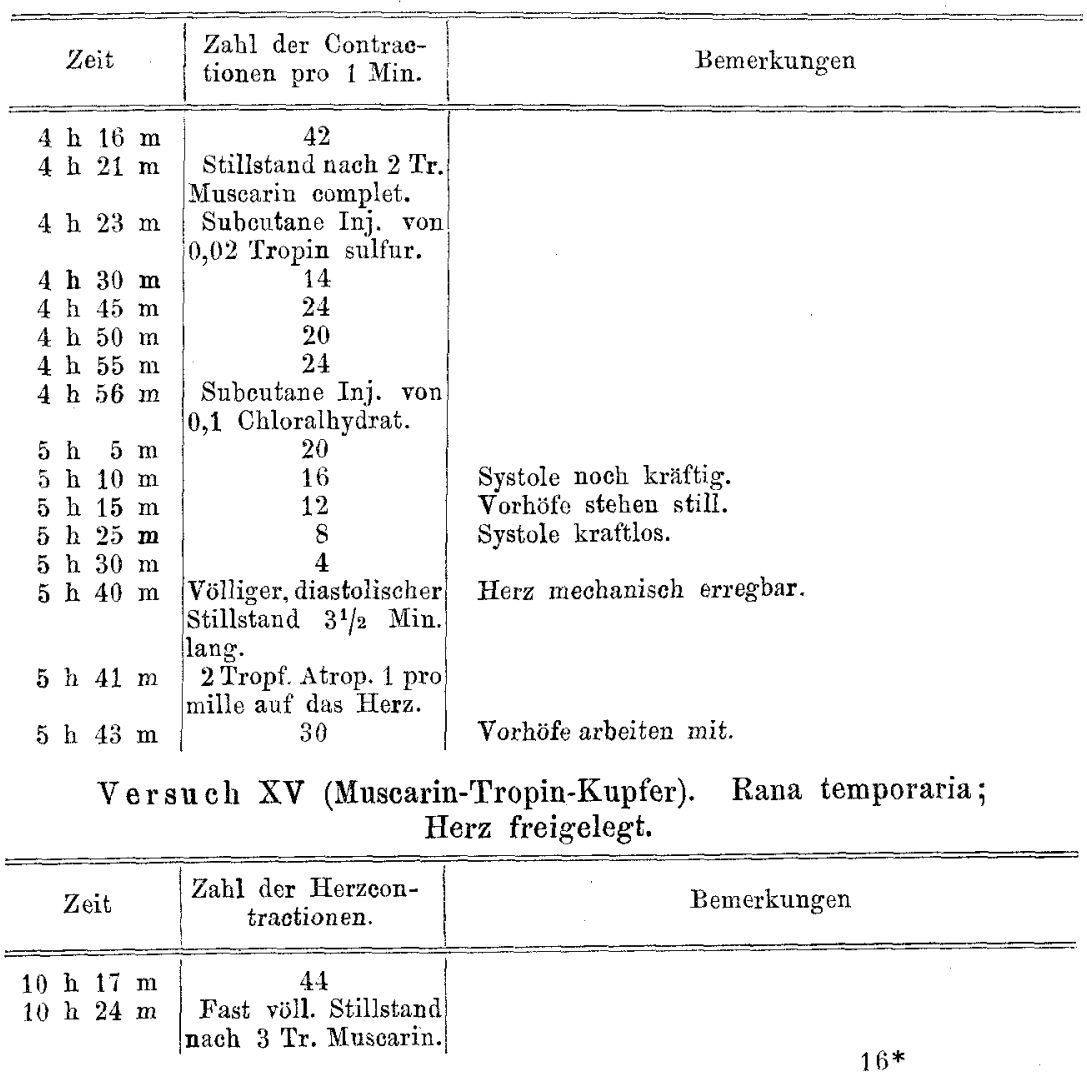




\begin{tabular}{|c|c|c|}
\hline Zeit & $\begin{array}{l}\text { Zahl der Herzcon- } \\
\text { tractionen }\end{array}$ & Bemerkungon \\
\hline $\begin{array}{llll}10 & \mathrm{~h} & 27 & \mathrm{~m} \\
10 & \mathrm{~h} & 30 & \mathrm{~m} \\
10 & \mathrm{~h} & 35 & \mathrm{~m} \\
10 & \mathrm{~h} & 40 & \mathrm{~m} \\
10 & \mathrm{~h} & 42 & \mathrm{~m} \\
10 & \mathrm{~h} & 50 & \mathrm{~m} \\
10 & \mathrm{~h} & 55 & \mathrm{~m} \\
11 & \mathrm{~h} & 5 & \mathrm{~m} \\
11 & \mathrm{~h} & 20 & \mathrm{~m} \\
11 & \mathrm{~h} & 30 & \mathrm{~m} \\
12 & \mathrm{~h} & - & \mathrm{m} \\
12 & \mathrm{~h} & 5 & \mathrm{~m} \\
12 & \mathrm{~h} & 8 \mathrm{~m} \\
12 & \mathrm{~h} & 9 & \mathrm{~m} \\
12 & \mathrm{~h} & 12 \mathrm{~m}\end{array}$ & \begin{tabular}{|c|} 
Subcutane Inj. von \\
0,02 Tropin sulfur. \\
10 \\
28 \\
26 \\
Subcutane Inj. von \\
26 \\
26 \\
28 \\
32 \\
Subcutane Inj. von \\
32 \\
12 \\
\\
13 peristalt. Con- \\
tractionen. \\
2 Tr. Atropin 1 pro \\
mille auf das Herz. \\
16
\end{tabular} & $\begin{array}{l}\text { Nur Ventrikel; Vorhöfe sind noch in Ruhe. } \\
\text { Kräftige Ventrikelcontractionen; Vorhöfe still. } \\
5 \mathrm{mg} \text { CuO als weinsaures Kupferoxydnatron. } \\
\text { Vorhöfe arbeiten schwach mit. } \\
\text { Systole ist weit weniger energisch. } \\
\text { Kraftlose Contractionen. } \\
\text { Ebenso. } \\
8 \mathrm{mg} \mathrm{CuO} \mathrm{als} \mathrm{weinsaures} \mathrm{Kupferoxydnatron.} \\
\text { Lähmung des Herzens deutlich. } \\
\text { Ventrikelcontractionen bei gleichzeitig } 32 \text { Con- } \\
\text { tractionen der Vorböfe. }\end{array}$ \\
\hline
\end{tabular}

Die am Froschberzen beobachteten Wirkungen lassen sich demnach wie folgt zusammenfassen:

1. Tropin und eine Anzahl seiner Ester heben den Muscarinstillstand nur unvollständig anf.

2. Tropin und Tropeïne sind Reizmittel für das Herz. Sie steigern die Neigung des Ventrikels, in Systole zu verbarren und regen das durch Kupfer gelähmte Herz zu energischeren Contractionen an. Eine nähere Analyse dieser Wirkung führt zu der Deutung, dass es sich um Steigerung der Erregbarkeit der motorischen Herzganglien handelt.

3. Die unvollständige Aufhebung des Muscarinstillstands durch Tropin und einzelne Tropeïne (Acetyltropin, Snccinyltropin) kann durch die Reizwirkung auf das Herz erklärt werden; die Annahme einer atropinartigen Läbmung der Hemmungsapparate erscheint nicht nothwendig. Andere Tropeine (Lactyltropin) stehen in der Mitte zwischen den erwäbnten und dem Atropin, indem neben der gleichen Reizwirkung auf das Herz auch eine Lähmung der Hemmungsapparate nach grösseren Gaben nachweisbar wird. Nur diese letzteren besitzen auch mydriatische Wirkung.

Bekanntlich hat eine lebhafte Discussion dariber stattgefunden, ob dem Atropin erregende Wirkungen auf das Herz zukommen; jedenfalls treten dieselben aber erst nach ungemein viel höheren Gaben als jenen ein, die die Hemmungsapparate bereits völlig lähmen. ${ }^{1}$ )

1) Harnack und Hafemann, Archiv f. exp. Path. u. Pharm. Bd. XVII. 


\section{Wirkung des Tropins und der Tropeïne auf den Kreislauf des Warmblüters.}

Die Versuche uber die Wirkung der Substanzen auf den Blutdruck und insbesondere über die Erregbarkeit des Vagus unter ibrem Einflusse wurden an Kaninchen und Katzen angestellt. Von den ersteren Versuchsthieren ist es bekannt, dass manche Individuen zu Versuchen tiber die Hemmungsapparate des Herzens wenig geeignet erscheinen, weil man öfters von vornherein beide Vagi nur schwach wirksam findet. Andreiseits kann wieder bei der Unempfindlichkeit des Kaninchens gegen Atropin die Wirkung des Giftes auf die Hemmungsapparate sehr fliuchtig sein und die Erregbarkeit des Vagus nach Injection von $1 / 2-1 \mathrm{cg}$ Atropin schon nach $10-20$ Minuten wiederkehren, ja in einzelnen Fällen geht die Unzugänglichkeit der Hemmungsapparate dem Atropin gegenüber so weit, dass allmählich bis $2 \mathrm{cg}$ Atropin sulf. in die Vene eingeführt werden müssen, bis die Reizbarkeit des Vagus erlischt. Es kann deshalb aus dem Wirksambleiben des Vagusreizes nach Einfuhrung eines Giftes nicht obne weiteres auf das Fehlen atropinartiger Eigenschaften gescblossen werden; vielmebr muss erst durch eine nachfolgende Atropininjection gepriuft werden, wie leicht die Hemmungsapparate in dem betreffenden Versuche der läbmenden Wirkung zugänglich sind. Es wurden deshalb nur solche Versuche als negativ in Bezug auf eine lähmende Wirkung auf die Hemmungsapparate angesehen, in denen die Vagusreizung bei gleichem Rollenabstand wie vor Injection des Giftes auch 3-5 Minuten nach der Injection nach derselben die gleiche Pulsverlangsamung hervorrief, nach weiterer Einfuhrung von $1 / 2-1 \mathrm{cg}$ Atropin aber sich völlig wirkungslos zeigte. Das grösste Interesse beanspruchte vor allem die Wirkung des Tropins selbst auf die Hemmungsapparate des Warmblüters.

Versuch XVI (Tropin sulfuric.). Kaninchen von $1850 \mathrm{~g}$ Art. carotis mit dem Manometer verbunden, Vagi angeschlungen.

\begin{tabular}{|c|c|c|c|}
\hline Zeit & 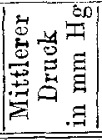 & 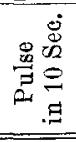 & Bemerkungen \\
\hline $\begin{array}{c}5 \mathrm{~h} 10 \mathrm{~m} \\
5 \mathrm{~h} 12 \mathrm{~m} \\
5 \mathrm{~h} 15 \mathrm{~m} \\
5 \mathrm{~h} 18 \mathrm{~m} \\
5 \mathrm{~h} 25 \mathrm{~m} \\
5 \mathrm{~h} 25 \mathrm{mbis} \\
5 \mathrm{~h} 32 \mathrm{~m} \\
5 \mathrm{~h} 33 \mathrm{~m}\end{array}$ & $\begin{array}{r}118 \\
80 \\
120 \\
48 \\
120 \\
- \\
130\end{array}$ & $\begin{array}{l}39 \\
40 \\
15 \\
40 \\
- \\
42\end{array}$ & $\begin{array}{l}\text { Rechter Vagus durchschnitten. } \\
\text { Rechter Vagus } 10 \text { Secunden bei Rollenabstand } 25 \mathrm{~cm} \text { gereizt. } \\
\text { Injection von } 0,05 \text { Tropin sulfuric. in } 2,5 \mathrm{~cm} \text { in die Vena } \\
\text { jugularis. }\end{array}$ \\
\hline
\end{tabular}




\begin{tabular}{|c|c|c|c|}
\hline Zeit & 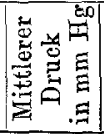 & 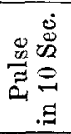 & Bemerkungen \\
\hline $\begin{array}{l}5 \mathrm{~h} 36 \mathrm{~m} \\
5 \mathrm{~h} 40 \mathrm{~m}\end{array}$ & $\begin{array}{r}90 \\
130\end{array}$ & $\begin{array}{l}16 \\
36\end{array}$ & Rechter Vagus 10 Secunden gereizt; derselbe Rollenabstand. \\
\hline $5 \mathrm{~b} 42 \mathrm{~m}$ & 80 & 14 & Rechter Vagus 10 Secunden gereizt; derselbe Rollenabstand. \\
\hline $5 \mathrm{~h} 45 \mathrm{~m}$ & 132 & 38 & \\
\hline $5 \mathrm{~h} 50 \mathrm{~m}$ & 128 & - & Injection von 0,005 Atropin sulfuric in die Vena. \\
\hline $51 \mathrm{~m}$ & 120 & 41 & \\
\hline $\begin{array}{l}5 \mathrm{~h} 54 \mathrm{~m} \\
5 \mathrm{~h} 60 \mathrm{~m}\end{array}$ & $\begin{array}{l}122 \\
165\end{array}$ & 40 & Rechter Vagus 10 Secunden lang; derselbe Rollenabstand. \\
\hline & & & Tod durch Erstickung des Thieres; Blutdruck steigt bis 165. \\
\hline
\end{tabular}

Versuch XVII (Tropin). Kaninchen $1700 \mathrm{~g}$. Art. carotis mit dem Manometer verbunden, Vagi angeschlungen, ohne Narkose.

\begin{tabular}{|c|c|c|c|}
\hline Zeit & 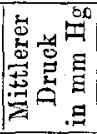 & 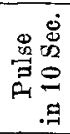 & Bemerkungen \\
\hline $\begin{array}{l}11 \mathrm{~h} 50 \mathrm{~m} \\
11 \mathrm{~h} 51 \mathrm{~m}\end{array}$ & $\begin{array}{r}108 \\
32\end{array}$ & $\begin{array}{l}46 \\
18\end{array}$ & $\begin{array}{l}\text { Linker Vagus durchschnitten und } 10 \text { Secunden gereizt; } \\
\text { Rollenabstand } 25 \mathrm{~cm} .\end{array}$ \\
\hline $\begin{array}{l}11 \mathrm{~h} 54 \mathrm{~m} \\
11 \mathrm{~h} 54 \mathrm{mbis} \\
11 \mathrm{~h} 58 \mathrm{~m}\end{array}$ & 100 & $\underline{42}$ & Einfuhrung von 0,03 Tropin sulfur in die Vena jugularis. \\
\hline $11 \mathrm{~h} 58 \mathrm{~m}$ & 90 & 36 & Dyspnoe. \\
\hline $11 \mathrm{~h} 59 \mathrm{~m}$ & 50 & 18 & Linker Vagus 10 Secunden gereizt; derselbe Rollenabstand. \\
\hline $12 \mathrm{~h} 4 \mathrm{~m}$ & 98 & 36 & Stärkere Dyspnoe. \\
\hline $12 \mathrm{~h} 5 \mathrm{~m}$ & 48 & 15 & Linker Vagus 10 Secunden gereizt; Rollenabstand der gleiehe. \\
\hline $\begin{array}{l}12 \mathrm{~h} 8 \mathrm{~m} \text { bis } \\
12 \mathrm{~h} 10 \mathrm{~m}\end{array}$ & 64 & - & Abermals 0,03 Tropin in die Vena. Absinken des Druckes. \\
\hline $12 \mathrm{~h} 15 \mathrm{~m}$ & 36 & 14 & Linker Vagus 10 Secunden gereizt; Rollenabstand $20 \mathrm{~cm}$. \\
\hline $\begin{array}{l}12 \mathrm{~h} 19 \mathrm{~m} \\
12 \mathrm{~h} 20 \mathrm{~m}\end{array}$ & 32 & 15 & $\begin{array}{l}\text { Ebenso. } \\
\text { Während der Injection von Atropin sinkt der Druck zur } \\
\text { Abscisse. }\end{array}$ \\
\hline
\end{tabular}

Tropin beeinflusste somit in diesen und anderen analogen Versuchen die Reizbarkeit der Vagusendigungen in Gaben bis $6 \mathrm{cg}$ nicht nachweisbar. Auch dieses Resultat des Warmblïterversuches scheint fur die den Froschherzversuchen gegebene Deutung zu sprechen, wenn auch bei dem enormen Unterschied in der Empfindlichkeit - 1/400 mgr Atropin sind im Stande, den Muscarinstillstand des Froschherzens aufzuheben, während Vagusreizung beim Kaninchen erst nach intravenöser Injection von $5 \mathrm{mgr}$ unwirksam wird - Schlüsse vom Warmblüterversuch auf das Froschherz immer unsicher bleiben.

Bei den untersuchten Tropeïnen ergaben die Blutdruckversuche, dass die Wirkung des Vagusreizes auf die Pulsfrequenz durch Acetyltropin und Succinyltropin nicht nachweisbar beeinflusst wird, während Hippuryltropin eine sehr geringe und rasch vorïbergehende Herabsetzung der Vaguswirkung hervorruft. Lactyltropin wirkt wie am 
Froschberzen in grossen Gaben atropinartig. Die Tropeïne stehen demnach in ihrer Wirksamkeit auf die Hemmungsapparate im Herzen in derselben Reihenfolge, in der sie sich nach der mydriatischen Wirkung ordnen, da auch an der Pupille Acetyl- und Succinyltropin unwirksam blieben und Lactyltropin deutliche Atropinwirkung zeigte. Einige Versucbsbeispiele mögen genügen.

Versuch XVIII (Succinyltropin). Kaninchen $1650 \mathrm{~g}$, Art. carotis mit dem Manometer verbunden, Vagi angeschlungen, ohne Narkose.

\begin{tabular}{|c|c|c|c|}
\hline Zeit & $\mid \begin{array}{cc}5 & 50 \\
0 \\
0\end{array}$ & 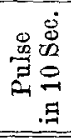 & Bemerkungen \\
\hline $4 \mathrm{~h} 21 \mathrm{~m}$ & 104 & 39 & \\
\hline $4 \mathrm{~h} 2$ & 63 & 14 & Rechter Vagus 10 Secund. lang gereizt; Rollenabstand $25 \mathrm{~cm}$. \\
\hline $4 \mathrm{~h} 24$ & - & - & 0,04 Succinyltropin hydrobromic. in die Vene eingefthrt. \\
\hline $4 \mathrm{~h} 32 \mathrm{~m}$ & & & Athmung hat ausgesetzt, künst. Respiration eingeleitet. \\
\hline $4 \mathrm{~h} 41 \mathrm{~m}$ & 90 & 36 & \\
\hline $4 \mathrm{~h} 42 \mathrm{~m}$ & 78 & 15 & Rechter Vagus 10 Sec. gereiat; derselbe Rollenabstand. \\
\hline $4 \mathrm{~b} 44 \mathrm{~m}$ & 94 & 36 & Athmet wieder selbständig. \\
\hline $4 \mathrm{~h}, \mathrm{~s}$ & 84 & $\begin{array}{l}15 \\
39\end{array}$ & Rechter Vagus 10 Seo. gereizt; der gieiohe Rollenabstand. \\
\hline 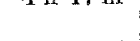 & 70 & 15 & Abermals rechter Vagus 10 Secunden gereizt. \\
\hline $4 \mathrm{~h} 50 \mathrm{~m}$ bis & - & - & 0,06 Succinyltropin in die Jugularis injicirt. \\
\hline $4 \mathrm{~h}:$ & 85 & 42 & Künstliche Respiration. \\
\hline $4 \mathrm{~h} 5$ & 80 & 20 & $\begin{array}{l}\text { Reizung des rechten Vagus } 10 \text { Sec.; der gleiche Rollen- } \\
\text { abstand. }\end{array}$ \\
\hline $4 \mathrm{~h} 58 \mathrm{~m}$ & 82 & 42 & 0,005 Atropin sulf. in die Vene. \\
\hline $5 \mathrm{~h} 2 \mathrm{~m}$ & 80 & 42 & Vagusreizung 10 Sec; derselbe Rollenabstand. \\
\hline
\end{tabular}

Versuch XIX (Hippuryltropin). Kaninchen $1700 \mathrm{~g}$, Art. carotis mit dem Manometer verbunden, Vagi angeschlungen, Urethannarkose.

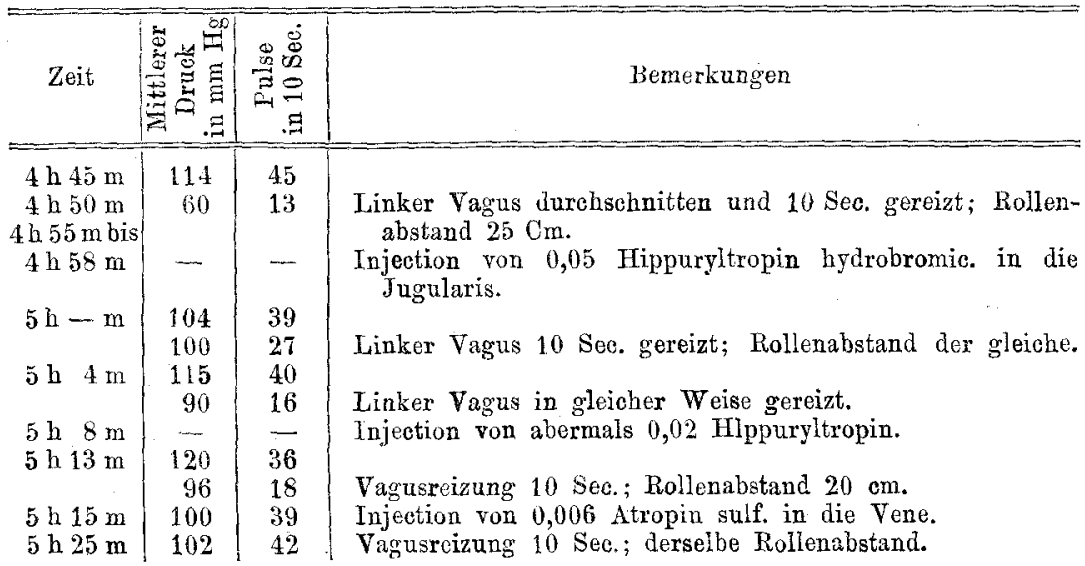


Versuch XX (Lactyltropin). Kaninchen $1800 \mathrm{~g}$, Art. carotis mit dem Manometer verbunden, Vagi angeschlungen.

\begin{tabular}{|c|c|c|c|}
\hline Zeit & $\mid \begin{array}{cc}3 \\
0 \\
0\end{array}$ & 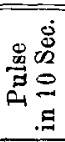 & Bemerkungen \\
\hline $4 \mathrm{~h} 37 \mathrm{~m}$ & $\begin{array}{r}110 \\
30\end{array}$ & $\begin{array}{l}48 \\
10\end{array}$ & $\begin{array}{l}\text { Reehter Vagus durchschnitten und } 10 \text { Sec. gereizt; Rollen- } \\
\text { abstand } 15 \mathrm{~cm} \text {. }\end{array}$ \\
\hline $\begin{array}{l}4 \mathrm{~h} 40 \mathrm{~m} \mathrm{bis} \\
4 \mathrm{~h} 46 \mathrm{~m} \\
4 \mathrm{~h} 48 \mathrm{~m} \\
4 \mathrm{~h} 50 \mathrm{~m}\end{array}$ & $\begin{array}{l}7 \\
110 \\
108 \\
108\end{array}$ & $\begin{array}{l}- \\
40 \\
40\end{array}$ & Einfuhrung von 0,1 Lactyltropin in die Vena jugularis. \\
\hline $\begin{array}{l}4 \mathrm{~h} 55 \mathrm{~m} \\
5 \mathrm{~h}-\mathrm{m}\end{array}$ & $\frac{110}{40}$ & 40 & $\begin{array}{l}\text { Rechter Vagus } 10 \text { Sec. gereizt; Rollenabstand } 12,5 \mathrm{~cm} \text {. } \\
\text { Injection ron Muscarin in die Vena jugularis setzt den } \\
\text { Blutdruck herab bis } \\
\text { derselbe bebt sich aber bald wieder auf. }\end{array}$ \\
\hline $5 \mathrm{~h}$ & 80 & 32 & Versuch abgebrochen. \\
\hline
\end{tabular}

In den angeführten Versuchen ist erwähnenswerth, dass auch bei jenen Tropeïnen, nach denen die Wirksamkeit des Vagus auf die Pulsfrequenz vollständig erhalten bleibt, der Blutdruck durch die Vagusreizung nicht mehr in demselben Maasse absinkt, als vor Einführung des Giftes; bei der gleichen Pulsverlangsamung durch den Vagusreiz sinkt der Blutdruck während derselben kaum mehr ab oder wenigstens weit weniger tief, als vor der Application des Giftes. Es liegt nahe, diese Erscheinung mit der in den Froschversuchen beobachteten Herzwirkung der Substanzen in Beziehung zu bringen; denn es wäre denkbar, dass die Reizwirkung der Tropeïne jener Abschwächung der Contractionen durch den Vagusreiz entgegenwirkte, die Ludwig und Coats ${ }^{1}$ ) zuerst am Froschherzen nachwiesen und die erst vor Kurzem $\mathrm{Cushny}^{2}$ ) auch in dem mit der Vagusreizung identischem Bilde der Muscarinvergiftung hervorgehoben hat. Es wurde dann bei gleicher Verlangsamung der Pulse die Contractionsenergie des einzelnen Herzschlags eine grössere sein, als vor der Einfübrung des Giftes, und sich dadurch auch das geringere Absinken des Blutdrucks erklären.

Acetyltropin und Lactyltropin bewirken ferner auch am Säugethiere eine deutliche Abnahme der Pulsfrequenz; bei den Froschherzversuchen wurde schon auf dasselbe Symptom hingewiesen. Die Abnahme der Pulsfrequenz tritt an Kaninchen und Katzen schon nach kleinen Gaben auf und ist von einer Vergrösserung der Pulselevation begleitet. Da erst nach sehr grossen Gaben dieser Tropeïne Symptome beginnender Herzläbmung eintreten, so kann die Pulsverlang-

1) Arbeiten aus der physiolog. Anstalt zu Leipzig 1869.

2) Archiv f. exp. Path. u. Pharm. Bd. XXXI. S. 432. 
samung keinesfalls auf eine Herabsetznng der Herzthätigkeit bezogen werden. Sie tritt auch an atropinisirten Thieren ein. Ueber ihre Ursache lässt sicb Näheres nicht aussagen, doch darf darauf hingewiesen werden, dass die Tropeïne dieses Symptom der Pulsverlangsamung mit einer Reihe anderer Reizmittel für das Herz gemeinsam haben, insbesondere mit Physostigmin und Campher. Vielleicht hängt die Abnahme der Pulsfrequenz und die Erhöhung der einzelnen Pulserhebungen mit einer der Wirkung am Froschberzen entsprechenden Veränderung der Herzeontraction zusammen. Eine Blutdrucksteigerung durch Beeinflussung der Herzthätigkeit lässt sich bei den Tropeïnen nicht nachweisen. Nimmt man aber eine erhöhte Erregbarkeit motorischer Herzganglien als Ursache der beobachteten Erscheinungen an, so fehlt es an Analogien für die Beurtheilung, ob eine solche Veränderung überhaupt zu erwarten wäre.

Durch grössere Gaben Acetyltropin, das wie Eingangs erwähnt central erregend wirkt, tritt allerdings erhebliche Blutdrucksteigerung ein. Dieselbe dürfte aber auf Reizung vasomotorischer Centren und nicht auf die Herzwirkung der Substanz zu beziehen sein, da sie am chloralisirten Thiere nicht zu beobachten war. An einer curarisirten und künstlich respirirten Katze trat so eine Anfangs anfallsweise Erhöhung des Blutdrucks bis $230 \mathrm{~mm} \mathrm{Hg}$ auf und hielt sich dann stundenlang auf dieser Höhe. Auch eine erregende Wirkung auf das Respirationscentrum kommt dem Acetyltropin zu und es könnte daran gedacht werden, das Atropin in seiner Anwendung als Erregungsmittel durch Acetyltropin zu ersetzen, dem die störenden Nebenwirkungen auf Pupille und Secretionen fehlen.

Die Abnahme der Pulsfrequenz bei gleichzeitiger Zunahme der Pulshöhe nach Substanzen, die wenigstens am Froschherzen als Erregungsmittel für das Herz angesprochen werden müssen, legten den Gedanken an therapeutische Versuche am Menschen nahe. Herr Prof. Vierordt hatte die Freundlichkeit, dieselben mit dem ungiftigsten der Tropeïne, mit Lactyltropin ausfubren zu lassen. Es ergab sich dabei, dass Gaben von etwa 0,01 Lactyltropin auch am Menschen deutliche Verlangsamung und Verstärkung des Pulses hervorriefen; da aber an Herzkranken öfters auch Arrhythmie des Pulses eintrat, so musste von weiteren Versuchen Abstand genommen werden.

Es wurden weiter auch einige Versuche über den Einfluss der untersuchten Substanzen auf die Secretionen angestellt. Um mich hierüber in einfacher Weise zu orientiren, rief ich an Kaninchen, die in geeigneter Weise in Seitenlage gefesselt waren, so dass der secernirte Speichel obne Verlust abfliessen musste, durch Pilocarpin Speichel- 
242 XIII. GotTlies, Ueber die Wirkungen des Tropins und der Tropeine.

fluss hervor und suchte den Ablauf der durch Pilocarpin hervorgerufenen Hypersecretion durch intravenöse Injection der Tropeïne $\mathrm{zu}$ beeinflussen. Es zeigte sich, dass von den untersuchten Substanzen höchstens das Lactyltropin in grösseren Gaben im Stande war den durch Pilocarpin erzeugten Speichelfluss zu hemmen, während kleine Atropingaben denselben stets aufheben. Das Lactyltropin schliesst sich demnach auch in seiner Wirkung auf die Secretionen dem Atropin an, wäbrend sich eine solche Wirkung bei den übrigen untersuchten Substanzen nicht nachweisen lässt.

Bei einem Vergleiche der peripheren Wirkungen der untersuchten Tropeïne fällt der Parallelismus dieser Symptome insbesondere in der Beeinflussung der Pupille und der Vagusendigungen im Herzen auf. Acetyltropin und Succinyltropin sind wie auch das Tropin selbst ohne Wirkung auf die Pupillenweite; sie lassen auch die Hemmungsapparate im Herzen unbeeinflusst. Lactyltropin dagegen, bei dem sich nach grösseren Gaben eine Lähmung der Vagusendigungen nachweisen lässt, ruft in concentrirterer Lösung auch am Auge Mydriasis hervor und bei dem gleichfalls schwach mydriatischen Hippuryltropin ist die Wirkung auf die Vagusendigungen beim Säugethier mindestens angedeutet. Auch dem Homatropin muss nach den Untersuchungen von Berthea $u^{1)}$ vaguslähmende Wirkung zugeschrieben werden. Wir sehen somit diese peripheren Atropinwirkungen gemeinsam auftreten oder gleichzeitig fehlen. Ebenso scheinen sich nach Versuchen von cand. med. Schiller im hiesigen Institute in dieser Beziehung die Scopoleïne zu verhalten.

Die Ergebnisse der Untersuchung lassen sich kurz dahin zusammenfassen:

1. Einzelne Tropeïne wirken nicht blos quantitativ verschieden von Atropin, sondern lassen dessen periphere Wirkungen gänzlich vermissen (Acetyltropin, Succinyltropin).

2. So weit die bisherigen Untersuchungen reichen, scheint in der Reihe des Tropins und der Tropeïne ein Parallelismus in der Beeinflussung der Vagusendigungen im Herzen und der Pupille zu bestehen. Diese Wirkungen können sowohl bei einzelnen Fettsäure-Estern auftreten (Lactyltropin), als auch bei aromatischen Tropeïnen fehlen.

3. Das Tropin selbst und die untersuchten, wenig giftigen Tropeïne sind Reizmittel für das Herz, während sich eine solche Wirkang bekanntlich beim Atropin nicht nachweisen lässt.

4. Diese Wirkung auf das Herz beruht wahrscheinlich auf einer Steigerung der Erregbarkeit motorischer Herzganglien und erklärt die Aufhebung des Muscarinstillstandes durch Tropin and jene Tropeïne, die nicht atropinartig wirken.

1) Berliner klinische Wochenschr. 1880. Nr. 41. 